\title{
Multimorbidity Trends in United States Adults, $1988-2014$
}

\author{
Dana E. King, MD, MS, Jun Xiang, MS, MA, and Courtney S. Pilkerton, MD, PhD
}

Importance: The simultaneous presence of multiple conditions in one patient (multi-morbidity) is a key challenge facing primary care.

Objective: The purpose of this study was to determine the prevalence of multi-morbidity and to document changes in prevalence during the last 25 years.

Design/Setting: Cross-sectional study using multiple years (1988-2014) of the National Health and Nutrition Examination Survey (NHANES) were analyzed.

Setting: Multiple years (1988 to 2014) of the National Health and Nutrition Examination Survey (NHANES) from the United States were analyzed.

Participants: Noninstitutionalized adults.

Main Outcomes and Measures: Number of chronic conditions per individual analyzed by age, race, gender, and socioeconomic factors.

Results: A total of 57,303 individuals were surveyed regarding the presence of multi-morbidity in separate surveys spanning 1988-2014. The overall current prevalence in 2013-2014 of $>2$ morbidities was $59.6 \%$ (95\% CI $58.1 \%-61.1 \%$ ), $38.5 \%$ had 3 or more, and $22.7 \%$ had 4 or more morbidities, which was significantly higher than in 1988 (45.7\%, 95\% CI 43.5\%-47.8\%, with >2 morbidities). Among individuals with 2 or more morbidities, $54.1 \%$ have obesity compared to $41.9 \%$ in 1988 . Among adults age $>65$, prevalence was $91.8 \%$ for 2 or more morbidities. Whites and Blacks had significantly higher prevalence $(59.2 \%$ and $60.1 \%)$ than Hispanic or "other" race $(45.0 \%, P<.0001)$. Women $(58.4 \%)$ had more current multi-morbidities $(>2)$ than men $(55.9 \%, P=.01)$.

Conclusions and Relevance: Multimorbidity is common and has been increasing over the last 25 years. This finding has implications for public health policy and anticipated health costs for the coming years. (J Am Board Fam Med 2018;31:503-513.)

Keywords: Cross-Sectional Studies, Health Care Costs, Health Policy, Multimorbidity, Nutrition Surveys, Prevalence, Primary Health Care, Socioeconomic Factors

The simultaneous presence of multiple conditions in one patient (multimorbidity) is a key challenge in primary care. Multimorbidity adds to the complexity of care and threatens the quality, coordination, continuity, and safety of care in the United States health care system and elsewhere. Despite the seriousness and far-reaching impacts of this phenomenon, characterization of this population in recent studies has focused on older populations, include a limited number of

This article was externally peer reviewed.

Submitted 8 January 2018; revised 3 April 2018; accepted 3 April 2018.

From Department of Family Medicine, West Virginia University, Morgantown, WV (DEK, JX, CSP).

Funding: Research reported in this publication was supported by the National Institute of General Medical Sciences of the National Institutes of Health under Award chronic conditions, and often do not include obesity as a chronic condition. ${ }^{1-8}$ The burden on patients with multimorbidity is considerable and is associated with increased mortality. ${ }^{9-12}$ A recent meta-analysis by Nunes and colleagues ${ }^{10}$ included 5806 multimorbidity studies, and mortality (26 studies were included) demonstrated a hazard ratio of 1.73 (95\% CI, 1.41-2.13) and 2.72 (95\% CI, 1.81-4.08) for people with 2 or more and 3 or more morbidities, respectively.

2U54GM104942-02. The content is solely the responsibility of the authors and does not necessarily represent the official views of the National Institutes of Health.

Conflict of interest: none declared.

Corresponding author: Dana E. King, MD, MS, Department of Family Medicine, West Virginia University, Morgantown West Virginia 26505 (E-mail: kingdana@ wvumedicine.org). 
In addition, heterogeneity in the included conditions of the studies was high, and obesity was not always included in the list of comorbidities, potentially underestimating the prevalence of multimorbidity. ${ }^{12-13}$ Including obesity in multimorbidity estimates is also crucial due to the well-studied link between obesity and a variety of complications, including diabetes, heart disease, cancer, and many others. ${ }^{14-19}$ Kivimaki and colleagues $^{20}$ have documented considerably increased cardiovascular events in obese versus nonobese cohorts in a pooled analysis of $16 \mathrm{co}-$ hort studies.

The purpose of this study was to determine the current prevalence of multimorbidity by using 11 common conditions, including obesity, and to examine trends in prevalence during the past 25 years. A secondary objective was to examine age, gender, race, and socioeconomic factors associated with multimorbidity prevalence.

\section{Methods}

\section{Study Population}

The National Health and Nutrition Examination Survey (NHANES) are serial, cross-sectional, stratified, multistage probability surveys designed to assess the health and nutrition status among the US civilian, noninstitutionalized population. The surveys were conducted by the National Center for Health Statistics, the data were collected on participants' demographic characteristics, nutrition, health, and diet through interviews in participants' homes, and medical examinations were conducted in a mobile examination center. All participants completed written informed consents, and protocols for conducting the NHANES study were approved by the Center for Disease Control and Prevention Institutional Review Board. Details on survey design and response rates can be found on the NHANES website. ${ }^{21}$

The present study combined NHANES III, which was conducted between 1988 and $1994,{ }^{22}$ and the continuous NHANES from 1999 to 2014, with data released in 2-year cycles. ${ }^{22}$

\section{Study Participants}

Participants aged 20 years or older, with nonzero weights (not nonrespondents) were included in the study sample. Of the 57,303 participants included in the study sample, there were 16,573 from
NHANES III and 40,730 from NHANES 1999 to 2014.

\section{Multimorbidity}

Multimorbidity, defined as the presence of 2 or more chronic conditions in a person, was the primary outcome of the study. Eleven chronic conditions were selected based on their clinical relevance and the availability of the NHANES data: cardiovascular disease, chronic obstructive pulmonary disease, chronic kidney disease, asthma, arthritis, cancer, stroke, hypertension, hyperlipidemia, diabetes, and obesity.

NHANES collects self-reports of diagnosis by a doctor for health conditions by asking a participant "have you ever been told by a doctor that you have that condition?" Participants were classified as having asthma, arthritis, stroke, and cancer, if the participant gave a positive answer to the question regarding these conditions. Participants were classified as having cardiovascular disease if they answered yes to having at least 1 of the following heart conditions: congestive heart failure, coronary heart disease, or heart attack. Although all 3 heart conditions were asked about in NHANES 1999 to 2014, participants in NHANES III were asked only about 2 of these conditions, congestive heart failure and heart attack. Participants were classified as having chronic obstructive pulmonary disease if they answered yes to having emphysema or chronic bronchitis.

Participants were classified as having hypertension, hyperlipidemia, or diabetes if they gave a positive answer to the self-reported question or had an individual medical measurement equal or greater than the recommended threshold. For example, a participant would be identified as having diabetes if he/she answered "yes" to the question regarding diabetes or had a measured hemoglobin A1c $\geq 6.5 \%$. The hemoglobin A1c cutoff was determined using the consistent standard set by the American Diabetes Association that is summarized in their clinical guidelines. ${ }^{23}$

Blood pressure cutoffs for hypertension were greater than $140 \mathrm{mmHg}$ for systolic blood pressure or $90 \mathrm{mmHg}$ for diastolic blood pressure. ${ }^{24}$ The cholesterol cutoff for determining hyperlipidemia was greater than $200 \mathrm{mg} / \mathrm{dL}$ of the total cholesterol, based on the Adult Panel III guidelines. ${ }^{25}$

Participants were classified as obese if they had a body mass index $\geq 30 \mathrm{~kg} / \mathrm{m}^{2}$. 
There was no self-report of diagnosis question for chronic kidney disease in NHANES. To identify participants with chronic kidney disease, we estimated level of kidney function from the estimated glomerular filtration rate (eGFR), which was calculated from recalibrated serum creatinine ${ }^{26}$ by using the Chronic Kidney Disease Epidemiology Collaboration creatinine equation. ${ }^{27}$ Specifically, eGFR $=141 \times$ min$(\mathrm{Scr} / \mathrm{k}, 1)^{\mathrm{a}} \times \max (\mathrm{Scr} / \mathrm{k}, 1)^{-1.209} \times 0.993^{\mathrm{Age}} \times 1.018$ [if female] $\times 1.159$ [if black]; with $\mathrm{Scr}=$ serum creatinine $(\mathrm{mg} / \mathrm{dL}) ; \mathrm{k}=0.7$, and $\mathrm{a}=-0.329$ if female; $\mathrm{k}=0.9$ and $\mathrm{a}=-0.411$ if male. As lower values of eGFR correspond with diminished kidney function, participants with a value of eGFR $<60 \mathrm{~mL} / \mathrm{min} /$ $1.73 \mathrm{~m}^{2}$ were identified as having chronic kidney disease.

Multimorbidity was categorized as $\geq 2$ morbidities, $\geq 3$ morbidities, and $\geq 4$ morbidities.

\section{Covariates}

Other demographic characteristics extracted for this study included age, gender, race, and socioeconomic status (education level, health insurance status, and ratio of family income to poverty). Age was divided into 3 groups: 20 to 44 years, 45 to 64 years, and 65 years or older. Race was combined into 4 groups of non-Hispanic white, non-Hispanic black, Hispanic, and other race. Participants' education level was grouped into 2 categories of " $<$ high school" and " $\geq$ high school." The ratio of family income to poverty was recoded as "above poverty" for greater or equal to 1.0 and "under poverty" for less than 1.0. Participants' health insurance status was defined as "yes" for having health insurance and "no" for not having health insurance.

\section{Statistical Analysis}

All data analyses were performed with SAS package version 9.3 (SAS institute Inc., Cary, NC). To account for the complex survey design (including oversampling), survey nonresponse, and poststratification, we incorporated appropriate sampling weights and SAS survey analysis procedures following NHANES analytic and reporting guidelines. ${ }^{28}$ Two year weights for NHANES 1999 to 2014 and 6-year weights for NHANES III were used for the prevalence estimate of individual cohorts. For trend analysis, we used the combined 6-year weights (NHANES III) and 16-year weight for NHANES 1999 to 2014. Missing data were assumed to be missing at random. To account for the confounding effect of age, age-standardized prevalence levels of multimorbidity ( $\geq 2$ morbidities, $\geq 3$ morbidities, and $\geq 4$ morbidities) were estimated and compared using $\mathrm{F}$ tests for overall samples and subsamples were stratified by NHANES cycles, race, gender, education level, health insurance status, and poverty level. The US 2010 Census population of adults aged 20 years or older was used for the calculation of the age-group weights (20 to 44 years, weight $0.5114 ; 45$ to 64 years, weight 0.3114 ; and 65 years or older, weight 0.1772$).{ }^{29}$ Logistic regression was performed to assess linear trends in levels of multimorbidity across NHANES cycles overall and by demographic and socioeconomic status. $P$ values for the trend analysis were calculated by regressing the levels of multimorbidity on the median year of the survey cycle. Statistical significance was determined if a 2 -sided $P$ value $<.05$.

\section{Results}

The prevalence of multimorbidity by demographic characteristics in NHANES 2013 to 2014 is presented in Table 1. Among the total sample of 5541 participants in the 2013 to 2014 cycle, 59.6\% (95\% CI, $58.1 \%-61.1 \%)$ had $\geq 2$ morbidities, $38.5 \%$ (95\% CI, 36.3\%-40.6\%) had $\geq 3$ morbidities, and $22.7 \%$ (95\% CI, $21.1 \%-24.3 \%$ ) had $\geq 4$ morbidities.

Compared with the groups aged 45 to 64 years and 20 to 44 years, the weighted prevalence of $\geq 2$ morbidities was higher in those aged 65 years or older (91.8\% vs $70.6 \%$ vs $37.5 \%, P<.0001)$. A similar significant difference between the age groups was also found in the prevalence of individuals with $\geq 3$ morbidities $(76.5 \%$ vs. $47.7 \%$ vs. $15.3 \%, P<.0001)$ and $\geq 4$ morbidities (55.6\% vs $26.4 \%$ vs $6.0 \%, P<.0001$ ).

There was higher age-standardized prevalence at all levels of multimorbidity in female participants than in male participants $(58.4 \%$ vs $55.9 \%, P=.01$ for $\geq 2$ morbidities; $38.4 \%$ vs $33.8 \%, P=.0002$ for $\geq 3$ morbidities; and $23.6 \%$ vs $18.4 \%, P<.0001$ for $\geq 4$ morbidities).

Across all 3 levels of multimorbidity, the agestandardized prevalence was consistently higher in non-Hispanic white and non-Hispanic black participants than Hispanic participants or participants of other race. The age-standardized prevalence of all levels of multimorbidity was similar among different education groups. Participants with health insurance had higher prevalence of $\geq 2$ and $\geq 3$ 


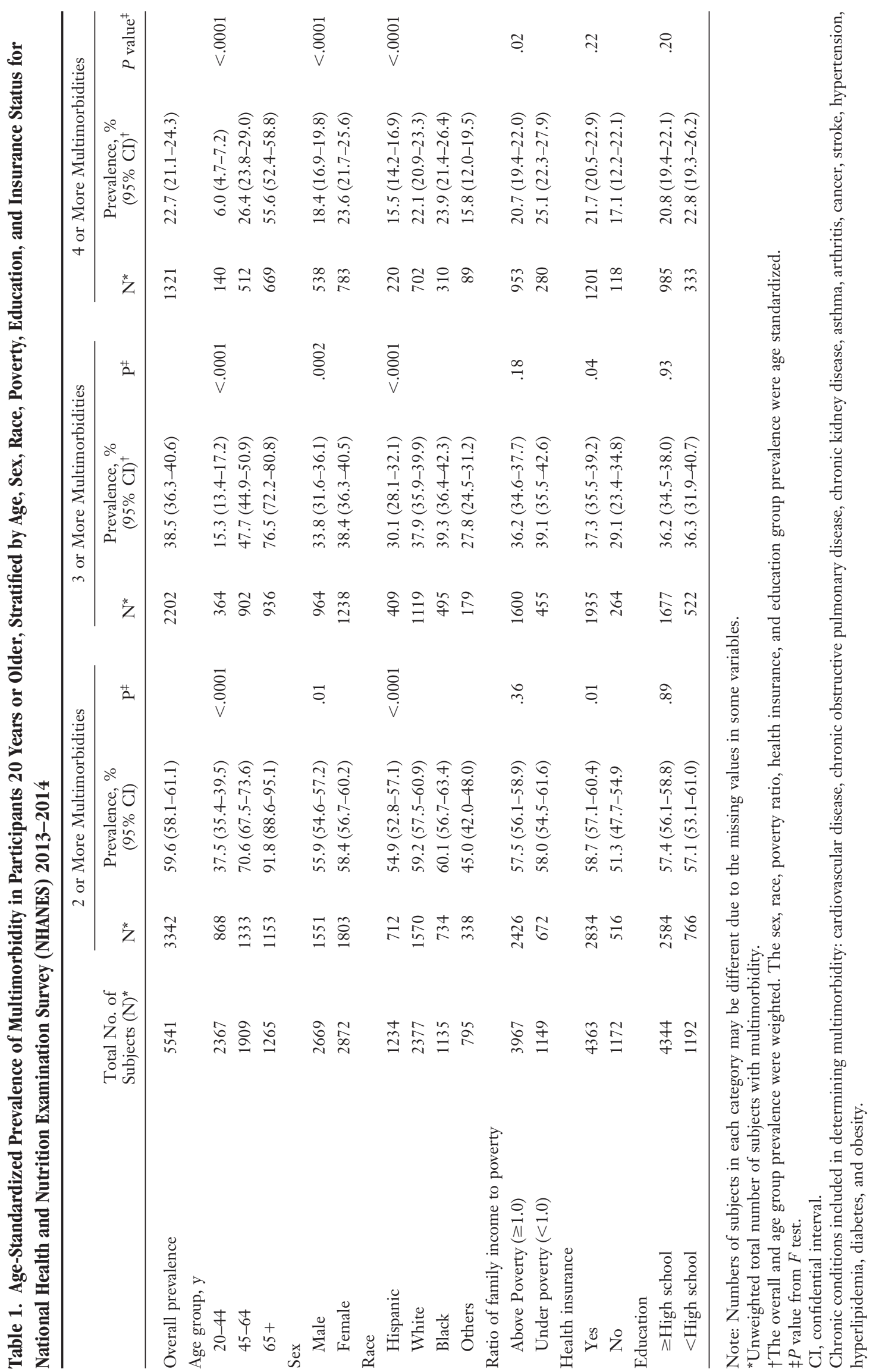


multimorbidities than their counterparts without health insurance. There was a lower prevalence of $\geq 4$ multimorbidities in participants "above poverty" compared with those in the "under poverty" group.

Tables 2, 3, and 4 summarize the trends in multimorbidity prevalence between 1988 and 2014 . The weighted overall prevalence of $\geq 2$ multimorbidities, $\geq 3$ multimorbidities, and $\geq 4$ multimorbidities significantly increased from $45.7 \%, 24.6 \%$, and $12.0 \%$ in 1988 to 1994 to $59.6 \%, 38.5 \%$, and $22.7 \%$ in 2013 to $2014(P<.0001$ for trend for all 3 levels) (as summarized in Figure 1). Significant increases in multimorbidity prevalence over the study period were seen in all levels of multimorbidity and for all age, gender, race, health insurance status, poverty level, and education level groups except other race. Although not significant, there was a decreasing trend in multimorbidity prevalence for other race.

Figure 2 illustrates the prevalence of each individual morbidity condition in the cohorts included in the study. Obesity experienced the largest increased trend of any condition across the study time frame $(P<.0001)$.

\section{Discussion}

The current findings document the high and growing prevalence of multimorbidity in adults in the United States. Overall, we observed over half of all adults $(59.6 \%)$ aged 20 and older have 2 or more multimorbidities, a proportion that has steadily increased from $45.7 \%$ in the 1988 to 1994 survey period. The prevalence was highest in people aged 65 years or older $(91.8 \%)$ and consistently higher in females than males.

These trend results are consistent with recent data from the Centers for Disease Control and Prevention showing a high prevalence of comorbidity in people with chronic conditions. For example, data from the National Health Interview Survey showed that 49\% of people with heart disease also had doctor-diagnosed arthritis. ${ }^{30}$ Recent Centers for Disease Control and Prevention data also showed that $25 \%$ of adults had at least 2 chronic conditions (out of 10 possible conditions). ${ }^{31}$ Dugolf and colleagues ${ }^{32}$ documented prevalence among Medicare beneficiaries and concluded that more than two-thirds of older adults have at least 2 chronic conditions.

The current study results show a higher prevalence than seen in other similar studies that is likely secondary to our selection of chronic conditions, notably obesity. Obesity is associated with a large number of pathologic processes and risks, including metabolic syndrome, vascular disease, cancer, oxidative stress, inflammation, as well as many others. Due to the considerable morbidity of obesity and its impact on a variety of health systems, we felt it was important to include it as a chronic condition rather than a control factor in the multimorbidity calculations for the current study. ${ }^{15-19}$

Similar to the current study's observation of increasing multimorbidity, this trend has been seen in other cohorts and other countries. Oostrum and colleagues $^{33}$ examined multimorbidity trends from 2001 to 2011 and saw increases in multimorbidity, but published much lower rates of multimorbidity (14.3\% to $17.5 \%, P<.01$ ), despite including 28 conditions seen in general practice. Their list included heart, lung, and mood disorders, as well as many others. A study in Canada by Pefoyo and colleagues $^{34}$ reported a multimorbidity rate that was increasing $(24.3 \%)$, but was still much lower than in the current study.

In US studies on a state level, similar patterns to the current study have been documented. Rocca and colleagues ${ }^{35}$ have studied a Minnesota cohort and reported similar trends for age and sex as in the current study.

Their overall rate of multimorbidity using 20 conditions was $77.3 \%$ for ages 65 years and older, compared with our finding of $91.8 \%$ in participants over 65 , but their study did not include obesity as one of the conditions.

The increase in multimorbidity over time suggests a worsening of the disease burden faced by individuals of all demographic characteristics. Over $91 \%$ of people over 65 are dealing with at least 2 serious chronic conditions or risk factors, and many are facing 4 or more. Prince and colleagues ${ }^{36}$ have recently reviewed the chronic disease burden among older people and concluded that it is a global problem and an epidemic. Furthermore, care of older adults with cardiovascular conditions is significantly complicated by the concurrent comorbidity burden that so frequently accompanies them. ${ }^{37}$

Possible explanations for the increasing prevalence of multimorbidity have been documented in the literature on numerous occasions, including unhealthy diet patterns, infrequent regular physical 


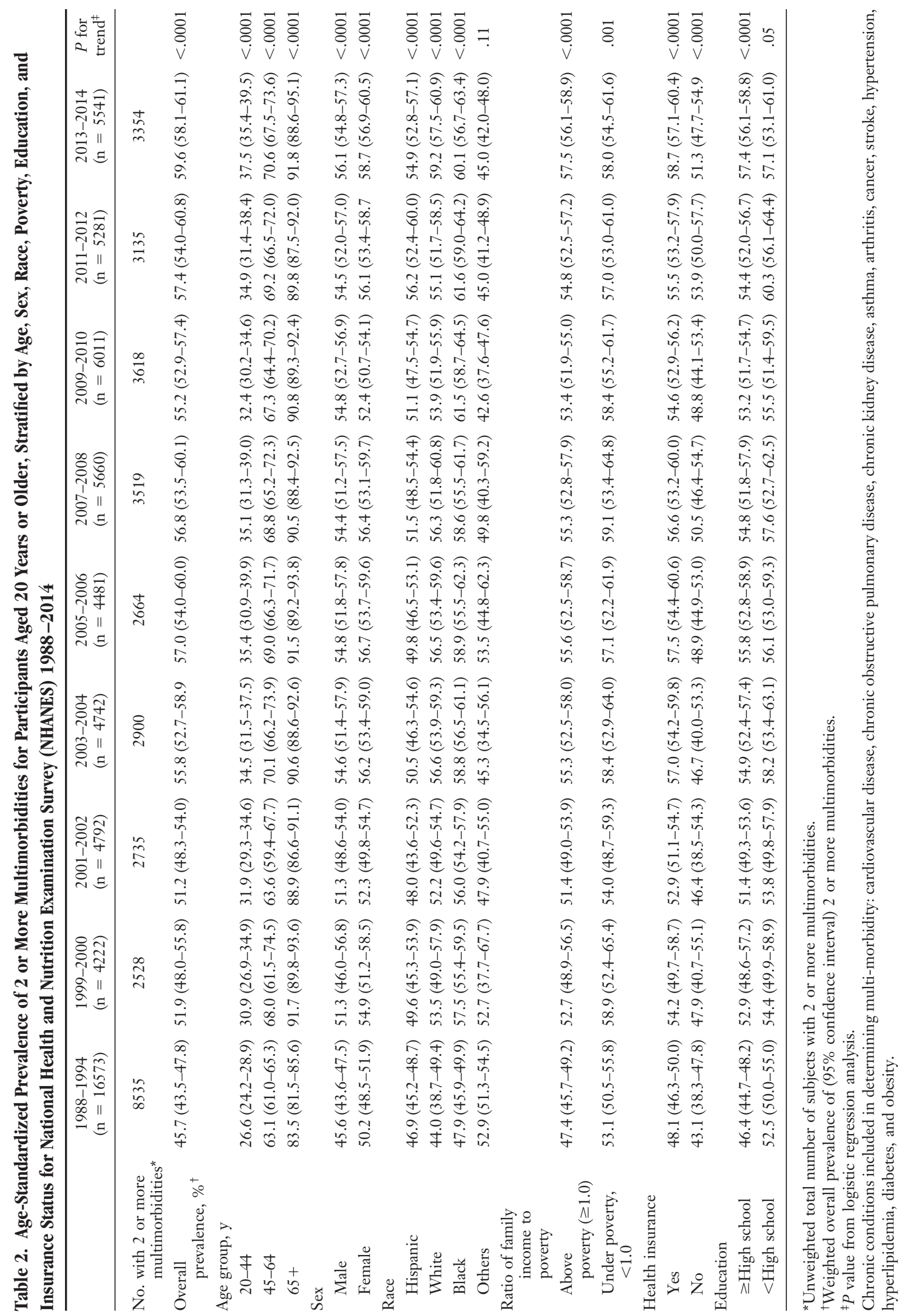



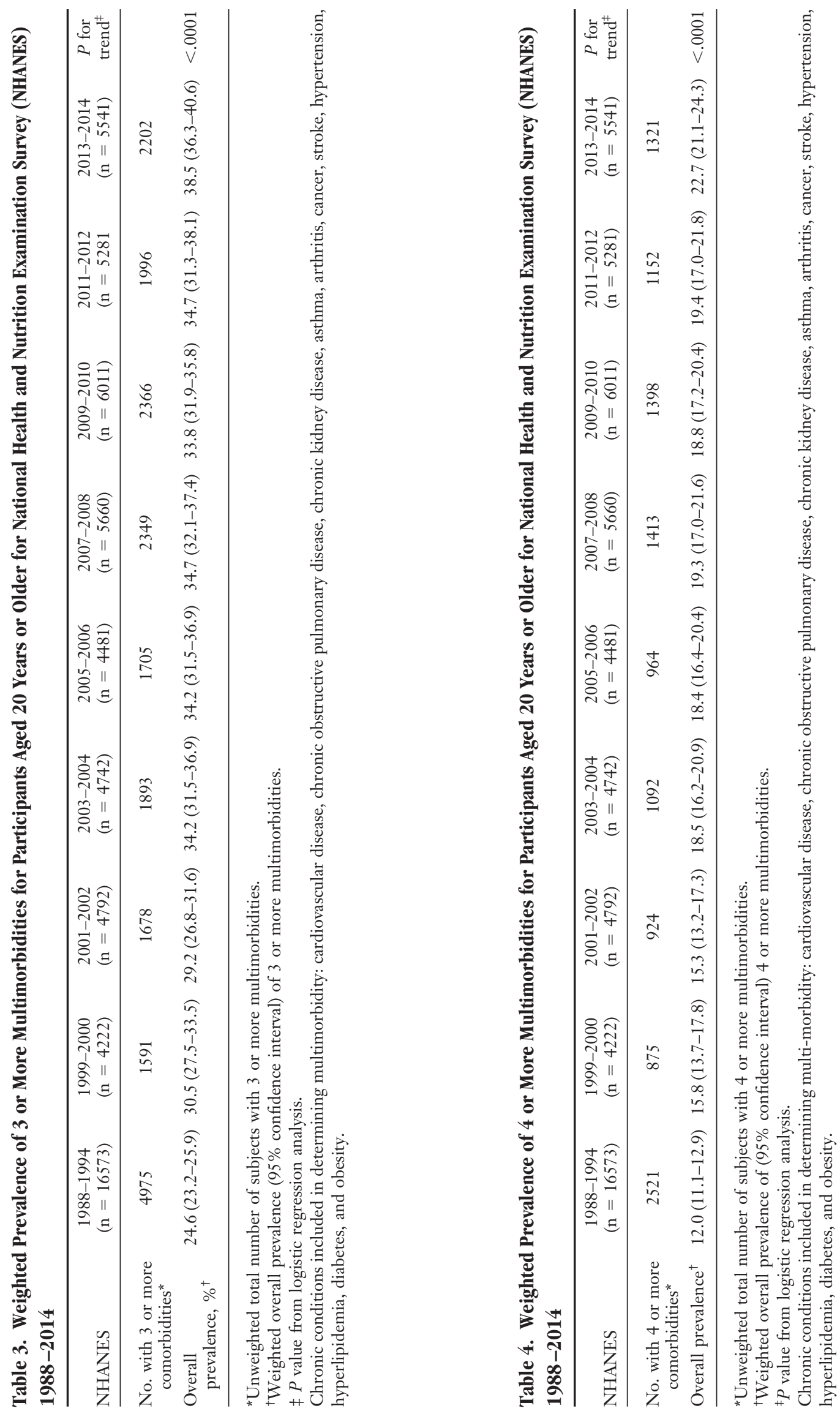

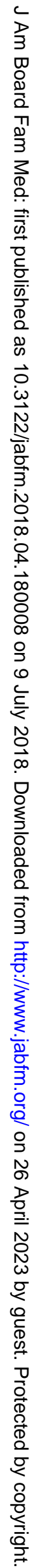


Figure 1. Age-standardized trends in multi-morbidity prevalence for participants 20 years or older from NHANES 1988-2014 by number of comorbidities.

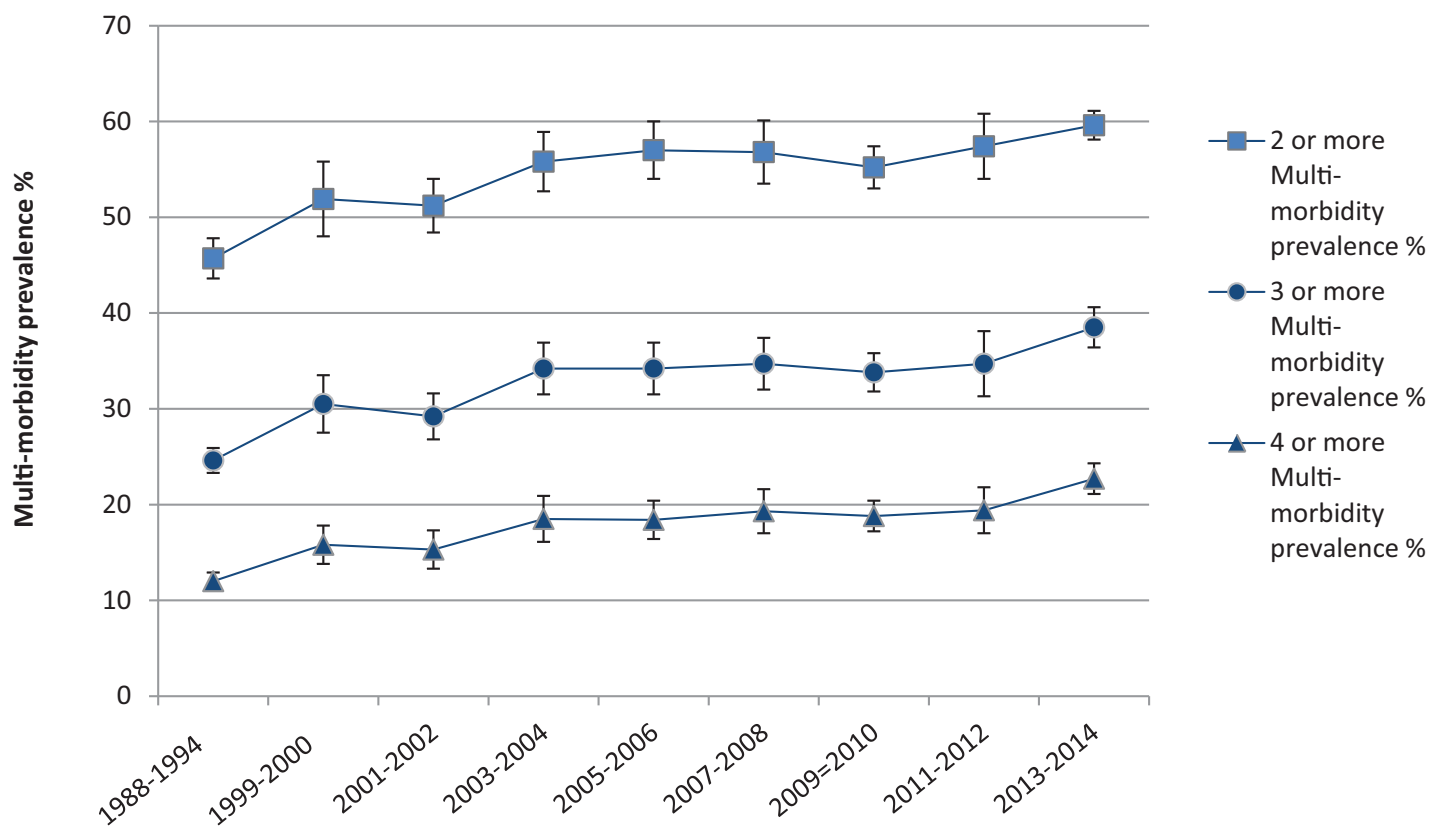

activity, smoking, and socioeconomic factors. ${ }^{38-44}$ creased diagnosis. ${ }^{45-46}$ The current study observed Other possible explanations are the prevalence of health disparities and the ease and regularity of access to primary care, which would lead to inthat much of the increasing trend in multimorbidity was likely due to the significant increase in obesity.

Figure 2. Age-standardized prevalence of various chronic conditions in participants 20 years or older from NHANES 1988-2014.

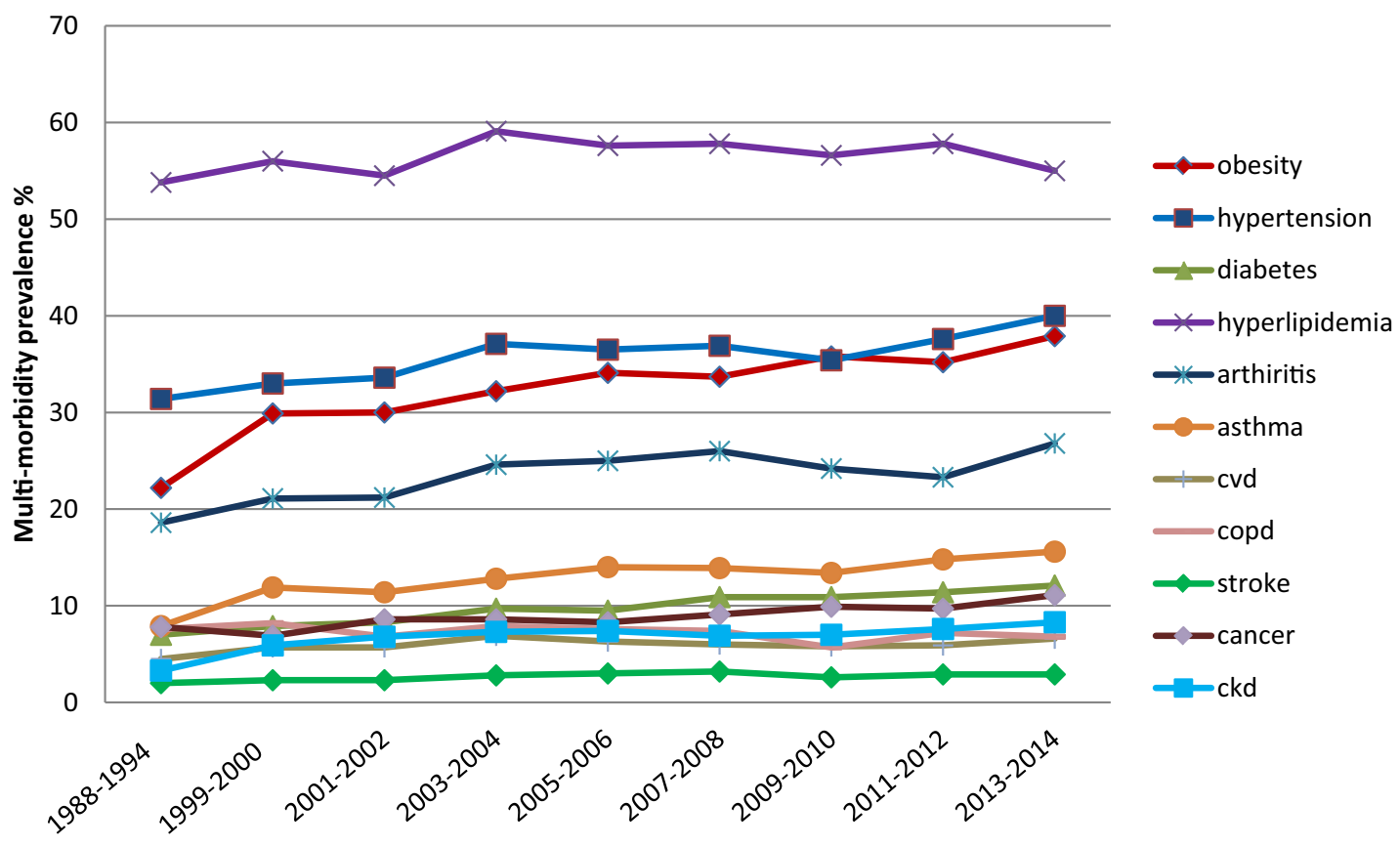


The association between trends and morbidities in people with insurance is complex and has been the subject of numerous studies, including 24 recent cross-sectional studies. ${ }^{47}$

Extensive further study will be needed to determine the roots of multimorbidity differences in populations and the impact on outcomes and disability. ${ }^{48}$

The association seen in the current study between having insurance and more comorbidities may be a consequence of several possible factors, including that insured people have easier health care access and may more frequently be told a diagnosis. Underdiagnosis of poorer individuals and uninsured populations also may be contributing. The local physical/geographic environment, insurance copays, regional variation, and many other factors may be contributing to this insurance socioeconomic equation and need further research.

This study has several limitations, including possible misclassification, consistency of data reported over cohort years, and cross-sectional data collection. Misclassification is a concern due to the reliance on self-reporting for the determination of several of the chronic conditions. Participants were considered to have the specific chronic condition by either a doctor-diagnosed history or by reaching the threshold for certain conditions, even if not formally diagnosed, such as blood pressure $>140 / 90$ or cholesterol $>200$. However, classification standards were consistently applied across the NHANES cohorts in the current study.

In addition, the comorbidities included in this study were limited because all selected conditions had to be included in each year cohort of the general NHANES questionnaire. For example, depression, anxiety, opioid addiction, and other mental health conditions known to be associated with morbidity have not been included consistently for all adult age groups in the NHANES cohorts over the period of this study, thus making it likely that we have underestimated multimorbidity. Specifically, opioid overuse or abuse data were not routinely collected even though it is recognized as a significant problem and growing contributor to premature mortality. ${ }^{49}$

Another limitation is that this study population consists of a series of cross-sectional studies, and, thus, the study is examining different people at each interval and does not represent the course of chronic disease in any individual.
In conclusion, multimorbidity for the 11 selected conditions is highly prevalent and has increased over the past 25 years. Obesity is a significant contributor to the trend. Public health leaders and policy makers should be attentive to these trends when designing policies and interventions to improve the public's health. Further research is needed to determine which interventions would be most helpful in addressing people with multimorbidity.

To see this article online, please go to: http://jabfm.org/content/ 31/4/503.full.

\section{References}

1. Jindai K, Nielson CM, Vorderstrasse BA, Quinones AR. Multimorbidity and functional limitations among adults 65 or older, NHANES 2005-2012. Prev Chronic Dis 2016;13:E151.

2. Lebwohl M. Does Treatment of psoriasis reduce cardiovascular comorbidities? J Invest Dermatol 2017;137:1612-3.

3. Mazarati AM, Lewis ML, Pittman QJ. Neurobehavioral comorbidities of epilepsy: role of inflammation. Epilepsia 2017;58:48-56.

4. Lehrke M, Marx N. Diabetes mellitus and heart failure. Am J Cardiol 2017;120:S37-S47.

5. Yin HL, Yin SQ, Lin QY, Xu Y, Xu HW, Liu T. Prevalence of comorbidities in chronic obstructive pulmonary disease patients: a meta-analysis. Medicine (Baltimore) 2017;96:e6836.

6. Dong Q, Liu H, Yang D, Zhang Y. Diabetes mellitus and arthritis: is it a risk factor or comorbidity?: a systematic review and meta-analysis. Medicine (Baltimore) 2017;96:e6627.

7. Radner H. Multimorbidity in rheumatic conditions. Wien Klin Wochenschr 2016;128:786-790.

8. Pefoyo AJ, Bronskill SE, Gruneir A, et al. The increasing burden and complexity of multimorbidity. BMC Public Health 2015;15:415.

9. Rosbach M, Andersen JS. Patient-experienced burden of treatment in patients with multi-morbidity- a systematic review of qualitative data. PLoS One 2017;12:e0179916.

10. Nunes BP, Flores TR, Mielke GI, Thume E, Facchini LA. Multimorbidity and mortality in older adults: a systemic review and meta-analysis. Arch Gerontol Geriatr 2016;67:130-8.

11. Landi F, Liperoti R, Russo A, et al. Disability, more than multimorbidity, was predictive of mortality among older persons aged 80 years and older. J Clin Epidemiol 2010;63:752-9.

12. Menotti A, Mulder I, Nissinen A, Giampaoli S, Feskens E, Kromhout D. Prevalence of morbidity and mulitmorbidity in elderly male populations and their impact on 10-year all-cause mortality: the 
FINE study (Finland, Italy, Netherlands, Elderly). J Clin Epidemiol 2001;54:680-6.

13. Chwastiak LA, Rosencheck RA, Desai R, Kazis LE. Association of psychiatric illness and all-cause mortality in the national department of veterans affairs health care system. Psychosom Med. 2010;72:817-22.

14. Censin JC, Nowak C, Cooper N, Bergsten P, Todd JA, Fall T. Childhood adiposity and risk of type 1 diabetes: a Mendelian randomization study. PLoS Med 2017;14:e1002362.

15. Reho JJ, Rahmouni K. Oxidative and inflammatory signals in obesity-associated vascular abnormalities. Clin Sci (Lond) 2017;131:1689-700.

16. King DE, Mainous AG 3rd, Matheson EM, Everett CJ. Impact of healthy lifestyle on mortality in people with normal blood pressure, LDL cholesterol, and C-reactive protein. Eur J Prev Cardiol 2013;20:73-9.

17. Engin A. The definition and prevalence of obesity and metabolic syndrome. Adv Exp Med Biol 2017; 960:1-17.

18. Tune JD, Goodwill AG, Sassoon DJ, Mather KJ. Cardiovascular consequences of metabolic syndrome. Transl Res 2017;183:57-70.

19. Lauby-Secretan B, Scoccianti C, Loomis D, Grosse Y, Bianchini F, Straif K. Body fatness and cancerviewpoint of the IARC working group. N Engl J Med 2016;375:794-8.

20. Kivimaki M, Kuosma E, Ferrie JE, et al. Overweight, obesity, and risk of cardiometabolic multimorbidity: pooled analysis of individual-level data for 120813 adults from 16 cohort studies from the USA and Europe. Lancet Public Health 2017;2:e277-e85.

21. National Center for Health Statistics. NHANES survey methods and analytic guidelines. Available from: https://wwwn.cdc.gov/nchs/nhanes/analytic guidelines.aspx. Accessed May 2018.

22. National Center for Health Statistics. NHANES analytic and reporting guidelines: the Third $\mathrm{Na}$ tional Health and Nutrition Examination Survey, NHANES III (1988-1994). Available from: http:// www.cdc.gov/nchs/data/nhanes/nhanes3/nh3 gui. pdf. Published October 1996. Accessed May 2018.

23. American Diabetes Association. Standards of medical care in diabetes-2018 abridged for primary care providers. Clin Diabetes 2018;36:14-37.

24. James PA, Oparil S, Carter BL, et al. 2014 evidencebased guideline for the management of high blood pressure in adults: report from the panel members appointed to the Eighth Joint National Committee (JNC 8). JAMA 2014;311:507-20.

25. Cho YK, Jung CH, Kang YM, et al. 2013 ACC/AHA cholesterol guideline versus 2004 NCEP ATP III guideline in the prediction of coronary artery calcification progression in a Korean population. J Am Heart Assoc 2016;5:e003410.

26. Selvin E, Manzi J, Stevens LA, et al. Calibration of serum creatinine in the National Health and Nutri- tion Examination Surveys (NHANES) 1988-1994, 1999-2004. Am J Kidney Dis 2007;50:918-26.

27. Levey AS, Stevens LA, Schmid CH, et al. A new equation to estimate glomerular filtration rate. Ann Intern Med 2009;150:604-12.

28. National Center for Health Statistics. Continuous NHANES web tutorial: specifying weighting parameters. Available from: https://www.cdc.gov/nchs/ tutorials/nhanes/SurveyDesign/Weighting/intro. htm. Updated May 2013. Accessed March 2018.

29. National Center for Health Statistics. Continuous NHANES web tutorial: age standardization and population counts. Available from: https://www. cdc.gov/nchs/tutorials/nhanes/NHANESAnalyses/ AgeStandardization/age_standardization_intro.htm. Updated May 2014. Accessed May 2018.

30. Ward BW, Schiller JS, Goodman RA. Multiple chronic conditions among US adults: a 2012 update. Prev Chronic Dis 2014;11:E62.

31. Centers for Disease Control and Prevention. Comorbidities. Available from: https://www.cdc.gov/ arthritis/data_statistics/comorbidities.htm. Updated February 2018. Accessed May 2018.

32. DuGoff EH, Canudas-Romo V, Buttorff C, Leff B, Anderson GF. Multiple chronic conditions and life expectancy: a life table analysis. Med Care 2014;52: 688-94.

33. Van Oostrom SH, Gijsen R, Stirbu I, et al. Time trends in prevalence of chronic diseases and multimorbidity not only due to aging: data from general practices and health surveys. PLoS One 2016;11: e0160264.

34. Pefoyo AJ, Bronskill SE, Gruneir A, et al. The increasing burden and complexity of multimorbidity. BMC Public Health 2015;15:415.

35. Rocca WA, Boyd CM, Grossardt BR, et al. Prevalence of multimorbidity in a geographically defined American population: patterns by age, sex, and race/ ethnicity. Mayo Clin Proc 2014;89:1336-49.

36. Prince MJ, Wu F, Guo Y, et al. The burden of disease in older people and implications for health policy and practice. Lancet 2015;385:549-62.

37. Krishnaswami A, Maurer MS, Alexander KP. Contextualizing myocardial infarction: comorbidities and priorities in older adults. Am J Med 2017;130: 1144-7.

38. Dieter BP, Tuttle KR. Dietary strategies for cardiovascular health. Trends Cardiovasc Med 2017;27: 295-313.

39. Shook RP, Hand GA, Drenowatz C, et al. Low levels of physical activity are associated with dysregulation of energy intake and fat mass gain over 1 year. Am J Clin Nutr 2015;102:1332-8.

40. Ali M, Li Y, O’Neal WT, Soliman EZ. Tobacco exposure as determined by serum cotinine and subclinical myocardial injury in individuals free from cardiovascular disease. Am J Cardiol 2017;120: $1114-7$. 
41. Amrock SM, Abraham CZ, Jung E, Morris PB, Shapiro MD. Risk factors for mortality among individuals with peripheral arterial disease. Am J Cardiol 2017;120:862-7.

42. Bruce MA, Martins D, Duru K, et al. Church attendance, allostatic load and mortality in middle aged adults. PLoS One 2017;12:e0177618.

43. King DE, Matheson E, Chirina S, Shankar A, Broman-Fulks J. The status of baby boomers' health in the United States; the healthiest generation? JAMA Intern Med 2013;173:385-6.

44. Fleisch Marcus A, Illescas AH, Hohl BC, Llanos AA. Relationships between social isolation, neighborhood poverty, and cancer mortality in a populationbased study of US adults. PLoS One 2017;12: e0173370.

45. Wang HH, Wang JJ, Lawson KD, et al. Relationships of multimorbidity and income with hospital admissions in 3 health care systems. Ann Fam Med 2015;13:164-7.

46. Chung RY, Mercer SW, Yip BH, et al. The association between types of regular primary care and hospitalization among people with and without multimorbidity: a household survey on 25,780 Chinese. Sci Rep 2016;6:29758.

47. Pathirana TI, Jackson CA. Socioeconomic status and multimorbidity: a systematic review and meta-analysis. Aust N Z J Public Health 2018;42:186-194.

48. Hilderink HB, Plasmans MH, Snijders BE, Boshuizen $\mathrm{HC}$, Poos MJ, van Gool CH. Accounting for multimorbidity can affect the estimation of the burden of disease: a comparison of approaches. Arch Public Health 2016;74:37.

49. Florence CS, Zhou C, Luo F, Xu L. The economic burden of prescription opioid overdose, abuse, and dependence in the United States, 2013. Med Care 2016;54:901-6. 